\title{
Rapid Spatial Mapping of Focused Ultrasound Fields Using a Planar Fabry-Pérot Sensor
}

\author{
Eleanor Martin, Edward Z. Zhang, James A. Guggenheim, Paul C. Beard, and Bradley E. Treeby, Member, IEEE
}

\begin{abstract}
Measurement of high acoustic pressures is necessary in order to fully characterize clinical high-intensity focused ultrasound (HIFU) fields, and for accurate validation of computational models of ultrasound propagation. However, many existing measurement devices are unable to withstand the extreme pressures generated in these fields, and those that can often exhibit low sensitivity. Here, a planar Fabry-Pérot interferometer with hard dielectric mirrors and spacer was designed, fabricated, and characterized, and its suitability for measurement of nonlinear focused ultrasound fields was investigated. The noise equivalent pressure (NEP) of the scanning system scaled with the adjustable pressure detection range between $49 \mathrm{kPa}$ for pressures up to $8 \mathrm{MPa}$ and $152 \mathrm{kPa}$ for measurements up to $25 \mathrm{MPa}$, over a $125 \mathrm{MHz}$ measurement bandwidth. Measurements of the frequency response of the sensor showed that it varied by less than $3 \mathrm{~dB}$ in the range 1-62 MHz. The effective element size of the sensor was $65 \mu \mathrm{m}$ and waveforms were acquired at a rate of $200 \mathrm{~Hz}$. The device was used to measure the acoustic pressure in the field of a $1.1 \mathrm{MHz}$ single-element spherically focused bowl transducer. Measurements of the acoustic field at low pressures compared well with measurements made using a Polyvinylidene difluoride needle hydrophone. At high pressures, the measured peak focal pressures agreed well with the focal pressure modeled using the Khokhlov-Zabolotskaya-Kuznetsov equation. Maximum peak positive pressures of $25 \mathrm{MPa}$ and peak negative pressures of $12 \mathrm{MPa}$ were measured, and planar field scans were acquired in scan times on the order of $1 \mathrm{~min}$. The properties of the sensor and scanning system are well suited to measurement of nonlinear focused ultrasound fields, in both the focal region and the low-pressure peripheral regions. The fast acquisition speed of the system and its low NEP are advantageous, and with further development of the sensor, it has potential in application to HIFU metrology.
\end{abstract}

Index Terms-Acoustic pressure measurement, Fabry-Pérot, field mapping, high-intensity focused ultrasound (HIFU).

\section{INTRODUCTION}

$\mathbf{T}$ HE measurement of high intensity focused ultrasound (HIFU) fields is critical in monitoring the stability of clinical ultrasound therapy systems, in validating models of ultrasound propagation, and in understanding these complex acoustic fields and their bioeffects. In order to fully characterize clinical therapeutic ultrasound fields, they must be measured at clinical levels where extremely high pressures

Manuscript received June 26, 2017; accepted August 31, 2017. Date of publication September 4, 2017; date of current version October 24, 2017. This work was supported in part by the Engineering and Physical Sciences Research Council under Grant EP/L020262/1 and in part by the European Union project FAMOS (FP7 ICT) under Contract 317744. (Corresponding author: Eleanor Martin.)

The authors are with the Department of Medical Physics and Biomedical Engineering, University College London, London WC1E 6BT, U.K. (e-mail: elly.martin@ucl.ac.uk).

Digital Object Identifier 10.1109/TUFFC.2017.2748886 of over $100 \mathrm{MPa}$ are generated in the focal region [1], [2]. In the ideal case, a suitable sensor for these conditions must be robust enough to withstand high pressures, temperatures, and cavitation, and have a high dynamic range $(\geq 60 \mathrm{~dB})$ and low noise equivalent pressure (NEP; $\lesssim 100 \mathrm{kPa}$ ) suitable for measuring the high-pressure focal region and low pressures elsewhere in the field. It must also have a wide bandwidth ( $>100-150 \mathrm{MHz}$ ) to capture high-frequency harmonics, and a small element size $(<\lambda / 4)$ to avoid spatial averaging in the narrow focal region and to provide an omnidirectional response. Ideally, measurements would also be acquired rapidly, to reduce uncertainty due to fluctuations in the experimental conditions. At present, there are no sensors that fulfill all of these criteria. In this paper, the usefulness of an optical sensor and scanning system in addressing these requirements is investigated and applied to the measurement of a nonlinear ultrasound field.

\section{A. Existing Sensors}

Currently available acoustic pressure sensors used for the measurement of ultrasound fields fall into two categories: piezoelectric sensors and optical sensors. Conventional piezoelectric hydrophones can be easily damaged by cavitation, heating, and direct mechanical effects. While purification and degassing of the test medium (usually water) can increase the threshold for cavitation, and use of a low duty cycle will reduce heating, damage is likely to occur eventually. One study has reported the use of a conventional Polyvinylidene difluoride (PVDF) membrane hydrophone for measurement of pressures up to peak positive and negative pressures of 27 and $10 \mathrm{MPa}$ before cavitation occurred, damaging the hydrophone [3]. However, exposing expensive hydrophones to pressures close to this level poses a significant risk, especially where water quality cannot be guaranteed. The pressure range of most piezoelectric sensors is also limited, often to 10 or $20 \mathrm{MPa}$, by the dynamic range of components such as preamplifiers. This limits them to the measurement of relatively low amplitude fields [4].

Small sensitive element sizes are desirable for measuring fields with high-frequency content. Although piezoelectric probe hydrophones with element sizes as small as $40 \mu \mathrm{m}$ are available, decreasing sensor size leads to a lower sensitivity and the frequency response of these types of hydrophone can be nonuniform [5]. Piezoelectric membrane hydrophones exhibit a more uniform frequency response but are only commercially available with $0.2 \mathrm{~mm}$ diameter elements at the smallest. Spatial averaging caused by relatively large 
element sizes can lead to significant underestimation of the peak positive focal pressure in highly nonlinear focused fields where the width of the focal peak may be less than $1 \mathrm{~mm}$ [1], [6].

Some piezoelectric hydrophones with extra coatings or layers added to increase their robustness have been reported in the literature. For example, a metal-coated piezoceramic needle hydrophone was reported capable of measuring up to $25 \mathrm{MPa}$ peak positive and $9 \mathrm{MPa}$ peak negative pressure before damage occurred [7], [8]. A PVDF membrane hydrophone with protective stainless steel and polymer coatings designed for use in HIFU fields is also available. Its use has been demonstrated for measurement of pressures up to approximately $60 \mathrm{MPa}$ [6]. By further adapting this design with a polyurethane backing layer, the measurement of pressures up to $75 \mathrm{MPa}$ peak positive and $15 \mathrm{MPa}$ peak negative was demonstrated, restricted by the operating limits of the transducer [3]. Both of these devices maintain low NEP of 45 and $65 \mathrm{kPa}$ for the two designs, respectively. These hydrophones have relatively large element sizes $(\geq 0.2 \mathrm{~mm})$ and somewhat limited bandwidths of $10 \mathrm{MHz}$ for the robust needle hydrophone and $40 \mathrm{MHz}$ for the robust membranes. The usable bandwidth of the robust membrane hydrophone was extended in this paper, however, by deconvolution of the full frequency response of the hydrophone [3]. Limited measurement bandwidth can lead to underestimation of the peak positive focal pressure if all harmonics are not captured [6].

Optical sensors such as the fiber optic hydrophone (FOPH) provide an alternative to the piezoelectric hydrophone, in general with the advantage of small element size and broad bandwidth [4], [9]. The Eisenmenger-type FOPH is based on pressure-induced refractive index changes at the tip of an optical fiber and has been used to measure upward of $100 \mathrm{MPa}$ peak positive pressure and $18 \mathrm{MPa}$ peak negative pressure [2], [10]. This is a suitable candidate for the measurement of HIFU fields due to its small size (100-125 $\mu$ m element size) and wide bandwidth $(\sim 100 \mathrm{MHz})$. It is also robust and has the advantage that the tip of the fiber can easily be recleaved to form a new fiber tip in the event of damage. However, its high NEP of more than 0.5 MPa [9], [11] (up to 2-3 MPa [6]) renders it unsuitable for mapping regions of lower pressure.

Higher sensitivity has been achieved with Fabry-Pérot polymer film ultrasound sensors [12], [13]. These sensors comprise a polymer film spacer sandwiched between a pair or mirrors and can be deposited on to either a planar substrate or the tip of an optical fiber. The exact construction of the mirrors, spacer, and substrate can be adjusted to optimize the sensitivity and bandwidth for a given application. For example, as part of the photoacoustic scanner described in [13], a sensor with a $22 \mu \mathrm{m}$ spacer had an NEP of $0.31 \mathrm{kPa}$ over a $20 \mathrm{MHz}$ measurement bandwidth, and $-3 \mathrm{~dB}$ bandwidth of $39 \mathrm{MHz}$. These NEPs are extremely low compared with the $50 \mathrm{kPa}$ NEP associated with a $75 \mu \mathrm{m}$ PVDF needle hydrophone [14]. While ideally suited to use in photoacoustic imaging when high sensitivity is required for the detection of low amplitude signals, this polymer spacer construction is not robust to high pressures and the upper limit of linear detection is low, typically a few megapascals.
To enable characterization of HIFU fields at both low and high outputs, a sensor that combines the individual benefits of the existing sensors in terms of robustness, small size, broad bandwidth, and low NEP is required. It may be possible to address these requirements using a Fabry-Pérot type sensor, by employing hard dielectric material to form the spacer rather than a polymer film. Previously, both fiber mounted and planar glass backed sensors of hard dielectric construction have been demonstrated [15], [16]. A planar sensor formed from $\mathrm{SiO}_{2}$ and $\mathrm{NbO}_{2}$ mirrors with a $\lambda / 2 \mathrm{SiO}_{2}$ spacer, $1.9 \mu \mathrm{m}$ thick in total, was shown to have a flat frequency response from 1 to $75 \mathrm{MHz}$, with a linear range of $37 \mathrm{MPa}$ [15]. The suitability of this type of sensor has been demonstrated for mapping nonlinear diagnostic ultrasound fields [17], and as a reference sensor for amplitude and phase calibration of hydrophones [18]. However, it has not previously been used for mapping HIFU fields.

\section{B. Field Scanning Techniques}

Currently, the most common method of acoustic field mapping is mechanical scanning of piezoelectric or FOPHs, which are commonly used for scanning at high output levels [1], [2], [19]. Measurement requirements are specified in the standards IEC 62127-1 [20] and IEC TS 62556 [21]. In general, an automatic positioning system is used to move a hydrophone in small steps through the acoustic field generated by a transducer mounted in a large tank of degassed and deionized water. Typically, the hydrophone is moved and waveforms are acquired with a frequency on the order of 1 scan point per second, with a spatial step size of less than half the acoustic wavelength. Consequently, it can take several hours to adequately sample the field of a typical HIFU transducer. Over these timescales, measurement errors are possible due to fluctuations in the source, hydrophone, and environmental conditions.

Other methods of acoustic field mapping have also been employed, which have some advantages in terms of acquisition speed and robustness. One approach to measuring high acoustic pressures, which removes the possibility of damaging sensors, is to use a noninvasive method. Historically, Schlieren imaging has been used to visualize ultrasound fields. Through projection images can be obtained in real time, but do not give quantitative information. More recently, a quantitative tomographic optical phase contrast measurement method has been described in [24]. However, due to difficulties with optical phase unwrapping, the acoustic pressure amplitude that can currently be measured using this approach is reported to be less than $10 \mathrm{MPa}$.

Planar Fabry-Pérot sensors [13] can also be used to make fast measurements of acoustic pressure. The sensors are interrogated by a scanning laser beam rather than mechanically scanning the sensor itself, enabling rapid data acquisition. These sensors have been extensively used in photoacoustic imaging [13], for ultrasonic field mapping [17], [25], [26], and for measurement of temperature and acoustic pressure for characterization of acoustic properties of materials [27]. 
a)

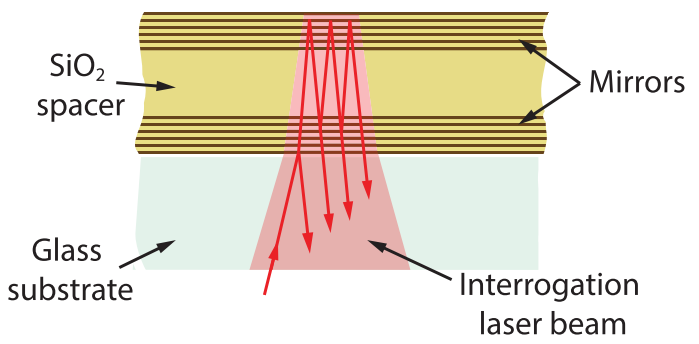

b)

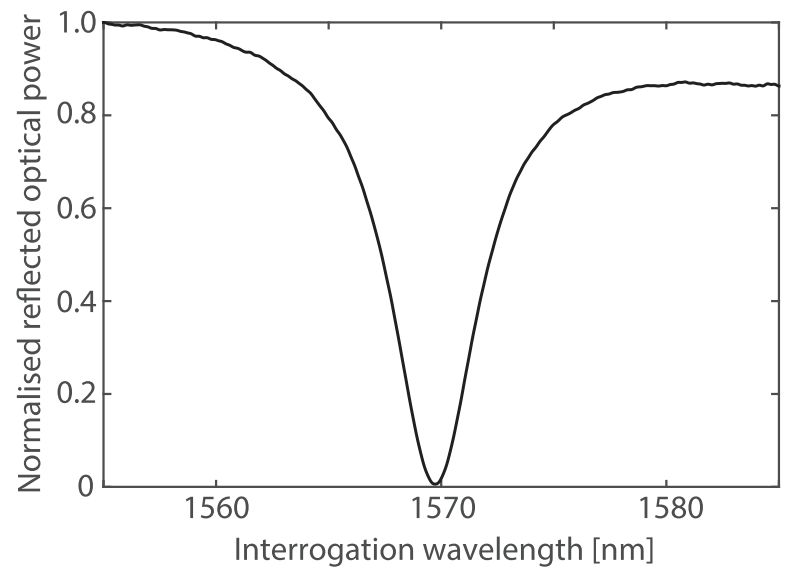

Fig. 1. (a) Schematic of the mirrors and spacer layers forming the FPI deposited on the glass substrate. The interferometer is interrogated by a scanned focused laser beam that is multiply reflected from the mirrors. (b) ITF measured for the sensor.

\section{Paper Outline}

In this paper, the design of a robust planar Fabry-Pérot sensor suitable for measurement of high acoustic pressures is discussed. This builds on the work reported in [15], using a similar sensor formed from all hard dielectric materials, but which has instead been designed to operate at $1550 \mathrm{~nm}$, coupled with a C-L (1516-1610 nm) band wavelength tunable laser and rapid scanning system. A full characterization of the sensor in terms of its frequency response, directivity, and NEP is presented in addition to a set of measurements of an HIFU field made with the sensor. Section II discusses the transduction mechanisms of the Fabry-Pérot sensor, and the construction of the sensor and scanning system. In Section III, the measured on-axis and directional frequency response and the NEP of the sensor are reported. Measurements of the field of a spherically focused ultrasound transducer at a range of drive levels are presented in Section IV.

\section{FABRY-PÉROT INTERFEROMETER}

\section{A. Transduction Mechanisms}

A Fabry-Pérot interferometer (FPI) consists of two plane parallel mirrors separated by a cavity or spacer. Incident light is multiply reflected from the mirrors and the multiple beams interfere at the first mirror [Fig. 1(a)]. The variation in optical power reflected from the FPI as a function of interrogation laser wavelength is termed the interferometer transfer function (ITF) and is shown in Fig. 1(b). The sensor is optimally biased by tuning the laser wavelength so that it corresponds to the maximum gradient of the ITF. Under these conditions, when the FPI is placed in an acoustic field, its optical thickness is modulated by the acoustic pressure causing a phase shift that results in a corresponding modulation of the optical power reflected from the sensor.

The overall sensitivity of a Fabry-Pérot sensor depends on both the optical phase sensitivity, which is the change in reflected optical power per unit phase shift, and the acoustic phase sensitivity, which is the optical phase shift per unit acoustic pressure [28]. The optical phase sensitivity depends on the power of the interrogating light and the reflectivity of the interferometer mirrors. The acoustic phase sensitivity is mainly governed by the optical and mechanical properties of the sensor. The incident pressure field induces small changes in the refractive index and thickness of the layers forming the mirrors and spacer, which modules the reflected optical power [16].

The reflectivity finesse of a sensor is defined as the ratio of the separation in wavelength of consecutive minima in the ITF to its full-width half-maximum. A very high finesse sensor has a steep ITF and is extremely sensitive to small optical phase changes generated by small changes in acoustic pressure, but as such has a very limited detection range.

As discussed in Section I-A, forming the interferometer from $\mathrm{SiO}_{2}$ hard dielectric mirrors and spacer provides the necessary robustness. However, the acoustic phase sensitivity is dependent on the refractive index and Young's modulus and will be reduced compared with the polymer-based sensors ( $n=1.444$ and $E=73 \mathrm{GPa}$ for $\mathrm{SiO}_{2}$ [30]; $n=1.65$ [13] and $E \sim 3 \mathrm{GPa}$ for Parylene C [31]). However, for measurement of high acoustic pressures, an increase in the upper limit of pressure detectable by the sensor is desirable and thus the decrease in acoustic phase sensitivity is acceptable. To offset the reduction in acoustic phase sensitivity, the optical phase sensitivity can be increased by increasing the reflectivity of the mirrors. The detection bandwidth of the sensor is related to the spatial variation of pressure across the interferometer and is therefore dependent on the thickness of the multilayer structure [32], with thinner sensors having a wider bandwidth.

\section{B. Sensor Construction}

As discussed in Section II-A, to increase the linear range and robustness of the sensor and maintain optical phase sensitivity, the sensor used in this paper was designed with an all hard dielectric construction. The mirrors were constructed from six sets of alternate layers of $\mathrm{SiO}_{2}$ and $\mathrm{ZrO}_{2}$ separated by a $3.9 \mu \mathrm{m} \mathrm{SiO}_{2}$ spacer. In this proof of concept design, all layers were deposited by electron beam-gun evaporation onto a $3 \mathrm{~mm}$ thick glass substrate. A parylene barrier coating was then applied to the sensor. This substrate was glued to another $16 \mathrm{~mm}$ thick glass plate to increase the time of arrival of acoustic reflections from the back of the substrate. The pressure reflection coefficient of the boundary between the two glass plates was measured to be less than 0.1 at $20 \mathrm{MHz}$, and lower at lower frequencies. In future designs, the interferometer structure will be deposited onto a single 
thicker substrate to provide the necessary time delay between the incident field and its reflection. The bottom face of the glass substrate was shaped into a wedge to eliminate parasitic optical reflections. The free spectral range of the sensor was $220 \mathrm{~nm}$ at an interrogation wavelength of $1569.5 \mathrm{~nm}$, the reflectivity finesse was 48.9 , and the visibility was 0.83 .

\section{Scanner System}

The scanning system was as previously described in [13]. To interrogate the sensor, a focused laser beam tunable in the range 1440-1640 nm (Tunics T100S-HP, Yenista Optics, France) was scanned across the sensor using a pair of orthogonal galvanometer mirrors (Edmund Optics, York, U.K.). The maximum scan area was $28 \mathrm{~mm} \times 30 \mathrm{~mm}$. The waveform acquisition rate was $200 \mathrm{~Hz}$, limited by the movement of the galvanometer mirrors and the tuning of the interrogation laser wavelength. To maximize the sensitivity of the sensor, the wavelength of the incident light is tuned to the maximum gradient of the ITF at each point. For the hard dielectric sensor, the thickness of the interferometer is very uniform and so the bias wavelengths lie within a range of approximately $1 \mathrm{~nm}$.

Fabry-Pérot sensors of both polymer and hard-dielectric construction have previously been shown to be temperature sensitive [12], [33]. Thermally induced changes in optical thickness cause changes in the reflectance of the sensor, resulting in a change in the dc voltage at the photodiode. In this paper, the sensor was not used to measure temperature. The low duty cycle and short pulse length employed in these measurements kept heating to a minimum and any temperatureinduced shifts in the ITF were within the linear range of the ITF. No change in sensitivity was observed following measurements.

At each scan point, the reflected light was measured by an InGaS photodiode [13], and the resulting voltage signal digitized by a digital storage oscilloscope and downloaded to a PC to build up a 2-D map of the time varying acoustic field distribution. The signals acquired at each scan point were corrected for the sensitivity of the interferometer by division by the maximum gradient of the ITF at that point on the sensor. The effective element size of the sensor is optically defined by the spot size of the incident laser light source $(65 \mu \mathrm{m})$ [13], which minimizes spatial averaging.

\section{SENSOR CHARACTERIZATION}

\section{A. Linearity and Sensitivity}

The linear range of the sensor was determined from the measured ITF shown in Fig. 1(b), and the pressure sensitivity of the system. The linear range of the sensor is defined as the range over which a straight line at a tangent to the point of maximum gradient (the bias point) of the measured ITF lies within $5 \%$ of the ITF. For the sensor used here, the linear range was $35 \mathrm{MPa}$ to within 5\% (allowing for the doubling in pressure at the sensor surface) and $56 \mathrm{MPa}$ to within $10 \%$. The dynamic range of the system was $49 \mathrm{~dB}$.

The NEP of the system was obtained as follows. First, the pressure sensitivity of the system in $\mathrm{mV} / \mathrm{MPa}$ was determined from the sensor output relative to a calibrated hydrophone using an incident acoustic wave emitted by a plane-piston transducer. Then the noise voltage was calculated as three standard deviations of the signal measured over a segment of the detected waveform in a time window immediately before the arrival of the acoustic signal. The NEP was then given by the ratio of the noise to the sensitivity. This was calculated over the full $125 \mathrm{MHz}$ measurement bandwidth used for measurements and over a $20 \mathrm{MHz}$ bandwidth for comparison with other sensors.

During measurements, the reflected interrogation light was measured by a photodiode that has a peak-to-peak saturation voltage of $5 \mathrm{~V}$. The measured voltage is proportional to the product of the sensitivity of the sensor, the interrogation laser power, and the acoustic pressure. It is therefore possible to adjust the measurement range of the system by changing the power of the interrogation laser. A low interrogation laser power is used for measuring high pressures and a high laser power is used to increase the signal-to-noise ratio (SNR) for measurement of low pressures. The NEP of the system scales with the interrogation laser power, as shown in Fig. 2(a). At the lower end of the pressure range (up to $8 \mathrm{MPa}$ ), the NEP over a $20 \mathrm{MHz}$ bandwidth is $26 \mathrm{kPa}$. This is lower than the $50 \mathrm{kPa}$ NEP associated with a similar sized $75 \mu \mathrm{m}$ PVDF needle hydrophone [14]. At the upper end of the linear range (56 MPa), the NEP is approximately $170 \mathrm{kPa}$ over a $20 \mathrm{MHz}$ bandwidth or $295 \mathrm{kPa}$ over a $125 \mathrm{MHz}$ bandwidth. This is lower than the NEP of the Eisenmenger-type FOPH that has an NEP of $500 \mathrm{kPa}$ or more [9], [11] but higher that the robust membrane hydrophones used to measure high amplitude acoustic fields [3].

\section{B. Frequency Response}

The relative normal incidence frequency response of the sensor was obtained by substitution calibration against a Fabry-Pérot sensor with a $20 \mu \mathrm{m}$ Parylene-C spacer and gold mirrors. The frequency response of this sensor was previously measured and modeled as described in [28] and had a $-3 \mathrm{~dB}$ bandwidth of $35 \mathrm{MHz}$; this will be herein referred to as the reference sensor. The sensor was mounted in the base of a water bath. An acoustic field was photoacoustically generated by laser light incident on a $2.5 \mathrm{~cm}$ diameter plane black absorber, which generated a planar monopolar acoustic pulse containing energy at frequencies up to approximately $80 \mathrm{MHz}$. The source was placed at a distance of $6 \mathrm{~mm}$ from the sensor and signals were acquired using the hard dielectric sensor from nine points at the center of the acoustic field distribution. At these positions in the acoustic field, the plane direct wave was temporally isolated from the edge waves, which were gated out, leaving only the plane wave. The sensor was then replaced by the reference sensor and the measurements were repeated. Signals were acquired with 2200 averages at each measurement point. The signals were then windowed using a $0.25 \mu \mathrm{s}$ Tukey window centered on the pulse, and their Fourier transforms were obtained. The magnitudes of the fast Fourier transforms (FFTs) were normalized and averaged. The frequency-dependent relative sensitivity of the hard dielectric 


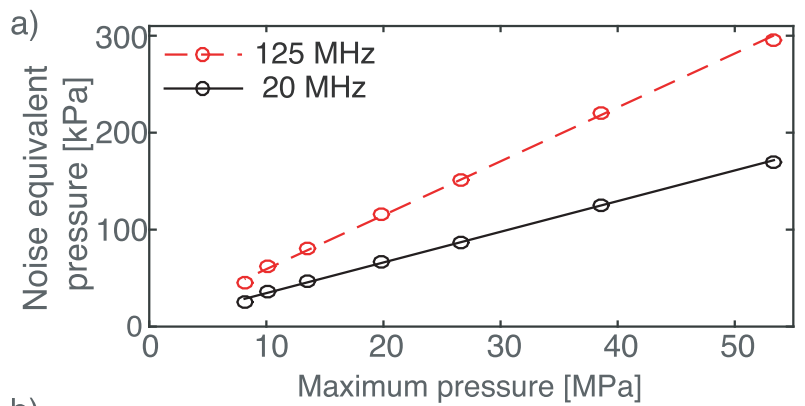

b)
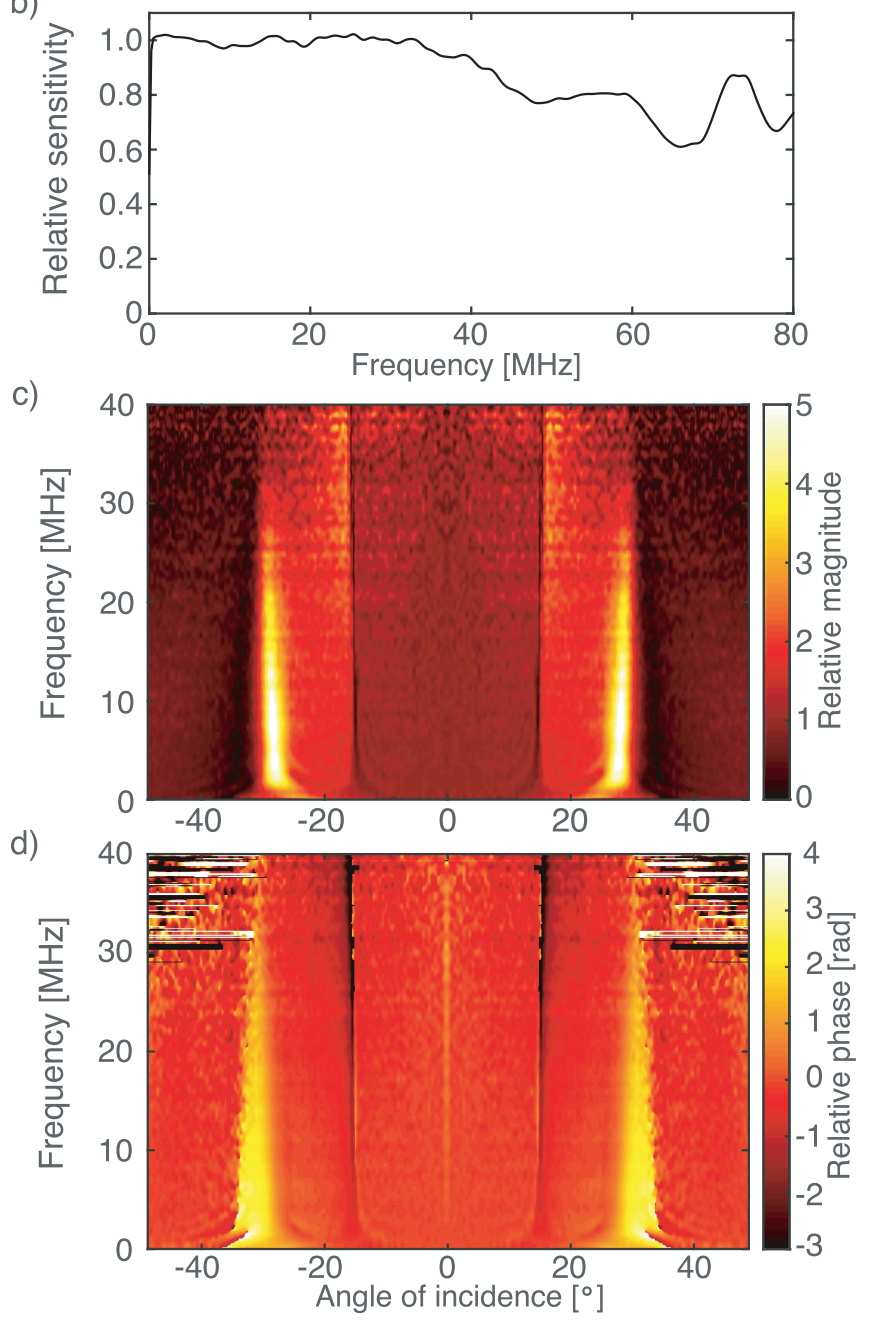

Fig. 2. (a) NEP of the system as a function of the upper limit of detectable pressure of the system. (b) Relative normal incidence frequency response of the sensor. (c) Magnitude of the directional response normalized to one at $\theta=0$. (d) Phase of the directional response referenced to zero at $\theta=0$.

sensor was then calculated by

$$
S_{\mathrm{HD}}(f)=S_{G}(f) \frac{\mathcal{F}_{\mathrm{HD}}(f)}{\mathcal{F}_{\mathrm{G}}(f)}
$$

where $S_{G}(f)$ is the sensitivity of the reference sensor, $\mathcal{F}_{\mathrm{HD}}$ is the Fourier transform of the signal acquired from the hard dielectric sensor, and $\mathcal{F}_{\mathrm{G}}$ is the Fourier transform of the signal acquired from the reference sensor. The relative frequency response of the sensor is shown in Fig. 2(b). The measured response was constant to within $1 \mathrm{~dB}$ in the range $1-40 \mathrm{MHz}$ and to within $3 \mathrm{~dB}$ up to $62 \mathrm{MHz}$. The fluctuations beyond this range may be due to the low SNR of the reference sensor signals due to its bandwidth ( $35 \mathrm{MHz}-3 \mathrm{~dB}$ bandwidth, sensitivity decreasing to a minimum at approximately $90 \mathrm{MHz}$ ). Previously, a planar $\mathrm{SiO}_{2}$ sensor of smaller thickness $(1.9 \mu \mathrm{m}$ in total) was shown to have a frequency response that was constant to within the calibration uncertainty in the range 1-75 $\mathrm{MHz}$ [15]. It may be expected that the frequency response of the sensor measured here would be similar in terms of its flatness, although with a reduced bandwidth given its larger thickness.

\section{Directional Response}

The directional response of the sensor was measured as described in [29], using the same broadband optically generated acoustic field as used to measure its frequency response. The source was mounted on a rotation stage to control the angle of incidence of the field on the sensor. The source was aligned such that its center of rotation was coincident with the sensor surface and the beam axis was aligned with the center of the sensor area. The source to sensor distance was approximately $30 \mathrm{~mm}$ to allow the lens tube holding the source to rotate within the walls of the tank. The angle of the source was stepped in $0.25^{\circ}$ increments from $-49^{\circ}$ to $+49^{\circ}$. At each angle, the signal was acquired with 20 averages with the pulse centered in a $2 \mu$ s window. A Tukey window the same length as the acquired data was applied to the signals and their Fourier transforms were acquired. The magnitudes of the spectra were normalized to the normal incidence values, and the unwrapped phase of the spectra was set to zero at normal incidence for each frequency. The measured data were then smoothed by fitting splines.

The magnitude and phase of the directional response of the sensor are plotted as a function of angle and frequency in Fig. 2(c) and (d), respectively. As Fig. 2 shows, the directional response of the sensor is not uniform with angle and several major features are observed. Below the critical angle for compressional waves at the water to glass interface $\left(\sim 15^{\circ}\right.$ assuming a speed of sound in water at $21{ }^{\circ} \mathrm{C}$ of $1488 \mathrm{~ms}^{-1}$ and a speed of sound in glass of $5640 \mathrm{~ms}^{-1}$ [30]), the magnitude and phase response are relatively flat. At the critical angle, there is a sharp increase in the magnitude response accompanied by a minimum in the phase response. There is a second sharp increase in the magnitude response, which coincides with the critical angle for shear waves at the water to glass interface $\left(\sim 27^{\circ}\right.$ assuming a shear speed of $3280 \mathrm{~ms}^{-1}$ [30]). The maxima and minima in the magnitude response are accompanied by rapid changes in the phase response. When the second critical angle is reached, other wave modes begin to influence the response. The exact nature of all wave modes involved is not currently known, and is the subject of ongoing work.

The complexity of the directional response of this sensor could give rise to errors in measurements of acoustic pressure, especially in focused and geometrically complex fields, as has been previously reported in [26]. For example, for the spherically focused transducer used in this paper, in the focal region, waves will be incident at angles up to $30.4^{\circ}$ (the angle 
subtended at the focus by the radius of the transducer), which exceeds both critical angles. A correction for the directional response is therefore necessary. This correction can be made by deconvolving the directional response from the signals measured over a 2-D plane

$$
P(x, y, t)=\mathcal{F}^{-1}\left(\frac{M_{\text {meas }}\left(k_{x}, k_{y}, \omega\right)}{D\left(k_{x}, k_{y}, \omega\right)}\right)
$$

where $\mathcal{F}^{-1}$ is the inverse Fourier transform, $M_{\text {meas }}\left(k_{x}, k_{y}, \omega\right)$ is the 3-D Fourier transform of the measured time-domain acoustic signals acquired over a planar area, $k_{x}$ and $k_{y}$ are the $x$ and $y$ wavenumber components, $\omega$ is the angular frequency, and $D\left(k_{x}, k_{y}, \omega\right)$ is the directional response of the sensor.

In practice, this correction is implemented as follows. The measured data are zero padded to achieve sufficient sampling in $\mathrm{k}$-space, as undersampling will result in an interpolation error when the correction is applied. The 3-D Fourier transform of the measured data is then calculated, along with the corresponding $k_{x}, k_{y}$, and $\omega$ values. Incidence angles for each wavenumber component are then calculated by

$$
\theta=\tan ^{-1}\left(\sqrt{\frac{k_{x}^{2}+k_{y}^{2}}{\frac{\omega}{c}^{2}-k_{x}^{2}-k_{y}^{2}}}\right) .
$$

Next, the directional response is interpolated onto the angle and frequency space occupied by the Fourier transform of the measured data. The correction is then applied by dividing the magnitude of the FFT of the data by the magnitude of the directional response and subtracting the phase of the directional response from the phase of the Fourier transform of the measured data. The magnitude and phase are then combined into a complex number, and the inverse Fourier transform is calculated.

In practice, deconvolution of the directional response from the measured signals introduces noise into the corrected time domain data. Just above the shear wave critical angle, the magnitude of the response is very low and so the SNR of the corresponding data will be very low. Applying the correction at these points is likely to introduce significant noise into the corrected data. To avoid this, the magnitude of the directional response correction $\left(1 /\left|D\left(k_{x}, k_{y}, \omega\right)\right|\right)$ was windowed to smooth these regions. A top hat window was applied, with a width determined by the angular position of the minimum in the correction at each frequency. For the correction at angles exceeding this, the correction was set to the value at the edge of the window.

\section{HIFU Field MEASUREMENTS}

\section{A. Ultrasound Source and Driving System}

To demonstrate the suitability of the hard dielectric FabryPérot sensor and rapid scanning system for ultrasonic field mapping, measurements of the field of a focused transducer were performed at a range of drive levels. The FP sensor, which had an area of $50 \mathrm{~mm} \times 30 \mathrm{~mm}$, was mounted in an aperture in the base of a specially designed water bath filled with deionized water, which was mounted above the optics necessary for interrogation of the sensor. The water was

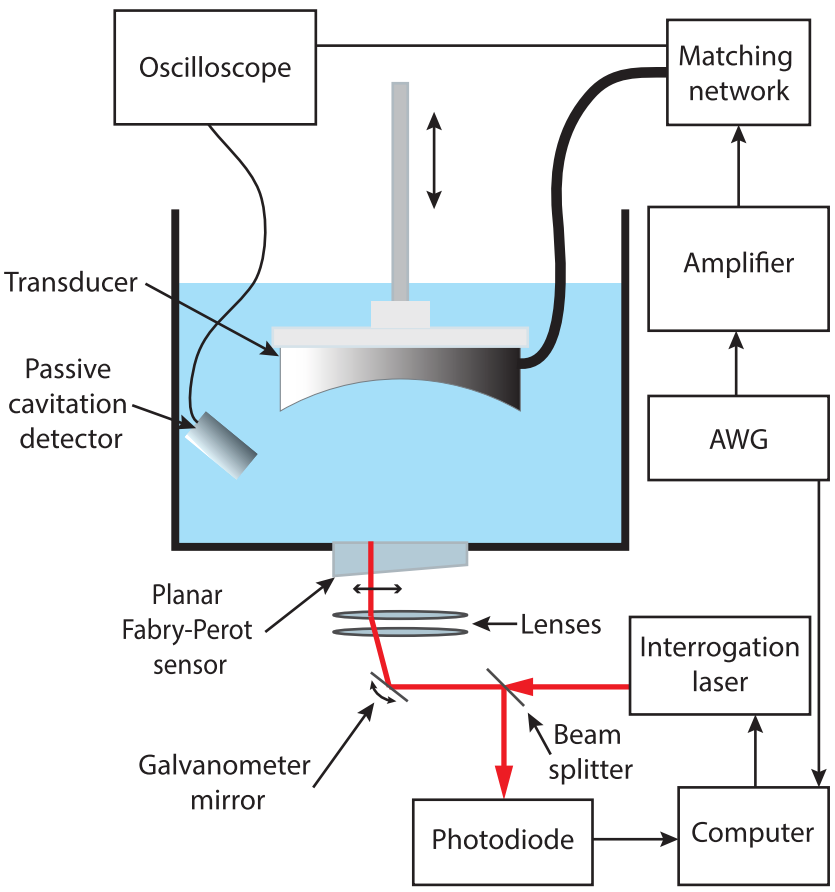

Fig. 3. Schematic of experimental configuration.

degassed by boiling to a dissolved oxygen concentration of approximately $2 \mathrm{mg} / \mathrm{L}$. Temperature control was provided by a polythene tube heat exchanger through which water was circulated from a thermostatically controlled water bath (Eco Silver RE 415S, Lauda, Germany). A diagram of the experimental arrangement is shown in Fig. 3. An acoustic field was generated by a single-element spherically focused bowl transducer (H101, Sonic Concepts, Bothell, WA, USA) mounted in the top of the water bath. The transducer had an active area diameter of $64 \mathrm{~mm}$ and focal length of $63.2 \mathrm{~mm}$ [22]. The transducer was driven with a four-cycle burst at $1.1 \mathrm{MHz}$. Input signals were generated by an Agilent 33522A Arbitrary Waveform Generator (Agilent, Berkshire, U.K.) before amplification by an E\&I A300 RF power amplifier (Electronics and Innovation Ltd., Rochester, NY, USA). This was coupled to the transducer via an impedance matching network. The transducer drive signal was monitored using an Agilent oscilloscope probe and Agilent DSO-X3204 oscilloscope (Agilent, as above). A $10 \mathrm{~mm}$ diameter $4 \mathrm{MHz}$ PVDF transducer was used for passive cavitation detection (the signal and spectrum were monitored using the oscilloscope).

\section{B. Comparison With Hydrophone Measurements at Low Pressure}

For comparison, measurements of the acoustic field were made at a low drive level with both the Fabry-Pérot sensor and a calibrated $0.2 \mathrm{~mm}$ PVDF needle hydrophone (Precision Acoustics Ltd., Dorchester, U.K.; hydrophone calibration provided by the National Physical Laboratory, Teddington, U.K.). Hydrophone measurements were performed in a tank of deionized water, with the hydrophone positioned using a scanning tank with five computer-controlled translation stages (Precision Acoustics, as above). The transducer was driven as 

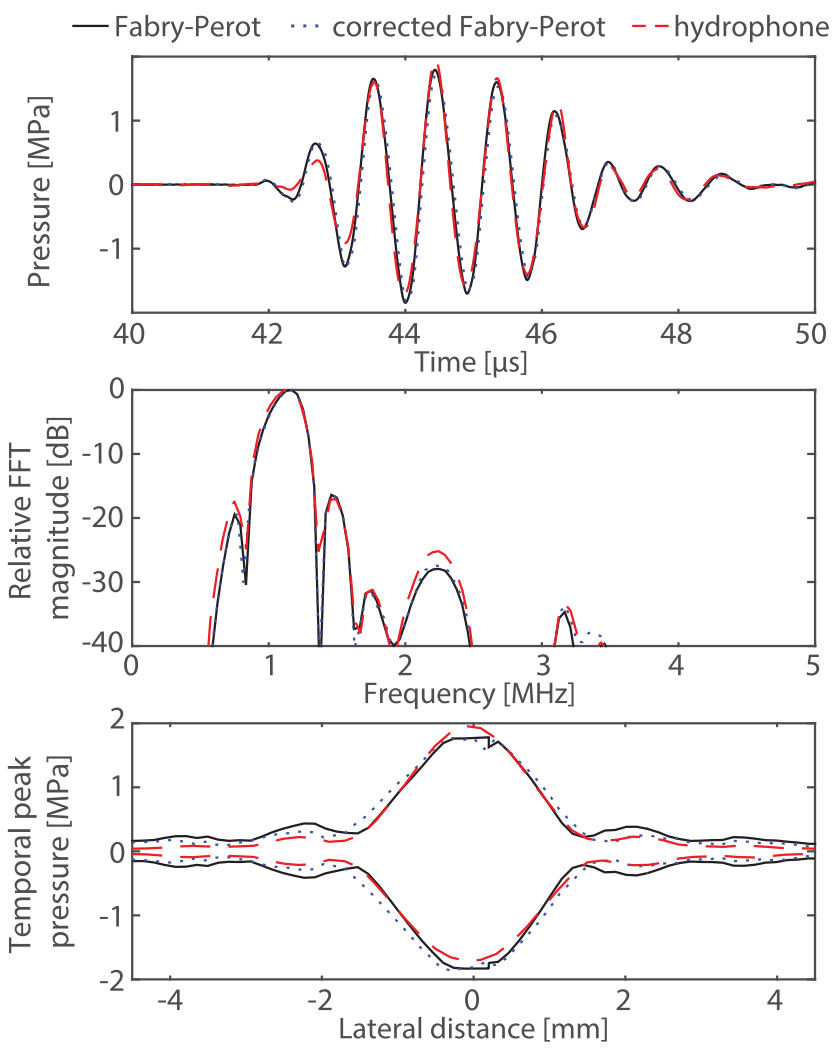

Fig. 4. Focal waveforms, spectra, and transverse beam profile of peak positive and peak negative pressure are shown for measurements made using a $0.2 \mathrm{~mm}$ PVDF needle hydrophone (red dashed line) and the Fabry-Pérot sensor. The black solid line shows the uncorrected Fabry-Pérot data and the blue dotted line shows the same data corrected for the directional response of the sensor.

described in Section IV-A with a peak-to-peak drive voltage of $36 \mathrm{~V}$. For the hydrophone measurements, the drive voltage was monitored using a Tektronix TPP0850 oscilloscope probe and Tektronix DPO5034B digital phosphor oscilloscope (Tektronix, U.K. Ltd., Berkshire, U.K.). The same oscilloscope was also used to digitize the hydrophone signal with a sampling frequency of $125 \mathrm{MHz}$ and 32 averages. Waveforms were then captured and stored via the scanning tank software. Axial and transverse line scans passing through the focus of the field were made with a step size of $0.2 \mathrm{~mm}$. A bandpass filter was applied to the signals $(0.35-20 \mathrm{MHz}$ passband) and the frequency-dependent sensitivity of the hydrophone was deconvolved from the measured waveforms to obtain the measured pressure.

A planar scan at the focal distance was then acquired with the Fabry-Pérot scanning system with 16 averages and a step size of $0.1 \mathrm{~mm}(45 \times 45$ points $)$. A line passing through the focal point of the field was then extracted from this measured plane. The signals were bandpass filtered (as above), and the measured directional response and frequency response of the sensor were deconvolved. The focal waveforms, spectra, and transverse peak positive pressure profiles are plotted for comparison in Fig. 4.

There are some differences between the beam profiles from the two sets of measurements. The shape of the main focal lobe is well captured by the Fabry-Pérot measurements, but there are discrepancies in the side lobes in the uncorrected Fabry-Pérot data. However, it can be seen that correcting for the directional response significantly reduces the differences in amplitude at the first side lobe to less than $10 \%$. The improvement is not as good in the second side lobe, most likely due to the windowing of the directional response that was applied to suppress noise in the corrected data. The windowing smooths the correction at angles of incidence above the shear wave critical angle of the water to glass interface, and therefore mainly affects measurement of pressure in the side lobes. The implementation of the directional response correction will be improved in future work.

\section{Measurement of HIFU Fields at Higher Drive Levels}

Two advantages of the Fabry-Pérot sensor are the speed with which planar scans can be performed (several orders of magnitude faster than a typical hydrophone scan) and the adjustable measurement range that enables the NEP to be minimized for measurements of both low and high pressures. To demonstrate these capabilities, measurements of the acoustic field were made at three different drive levels (peakto-peak voltage): 33,160 , and $280 \mathrm{~V}$. These correspond to pressures at the source of 43,211 , and $367 \mathrm{kPa}$, respectively. An area of $9 \mathrm{~mm} \times 9 \mathrm{~mm}$ was scanned with a step size of $50 \mu \mathrm{m}(180 \times 180$ points) in single acquisition mode (no averaging). The acquisition time was approximately $3 \mathrm{~min}$. The waveforms were high-pass filtered at $0.35 \mathrm{MHz}$ and corrected for the measured frequency response of the sensor. The waveforms were extracted from the center of mass of the field distribution, and the temporal peak pressure values were extracted from the waveforms measured along a line passing through this point. Fig. 5 shows focal waveforms, spectra, and temporal peak pressure profiles at the three transducer drive levels. The focal waveform becomes increasingly nonlinear as the transducer drive level is increased, with the amplitude of harmonics in the spectrum increasing. The peak positive pressure profile becomes increasingly narrow as the peak pressure increases, while the peak negative pressure profile broadens. The small effective element size of the system (65 $\mu \mathrm{m})$ limits spatial averaging, which can cause underestimation of the spatial peak pressure in the narrow focal region.

\section{Verification of Focal Pressure}

In order to verify the acoustic pressures measured at high transducer drive levels, the field was simulated in water using the Khokhlov-Zabolotskaya-Kuznetsov (KZK) equation. Simulations were performed using the Texas KZK code [34], [35]. The temporal step size was $\Delta \tau=0.0664(\Delta t=0.6 \mathrm{~ns}$, 1500 steps per cycle), the lateral spatial step size was $\Delta \rho=0.003(\Delta r=97 \mu \mathrm{m})$, and the axial spatial step size was $\Delta \sigma=2 \times 10^{-4}(\Delta z=25 \mu \mathrm{m})$.

The waveform used as the source boundary condition was obtained from near-field hydrophone measurements using linear acoustic holography as described in [22]. Briefly, the transducer was driven as described in Section IV-A, with a peak-to-peak drive voltage of $7.3 \mathrm{~V}$. The pressure was measured on a lateral plane in the near field of the transducer, 

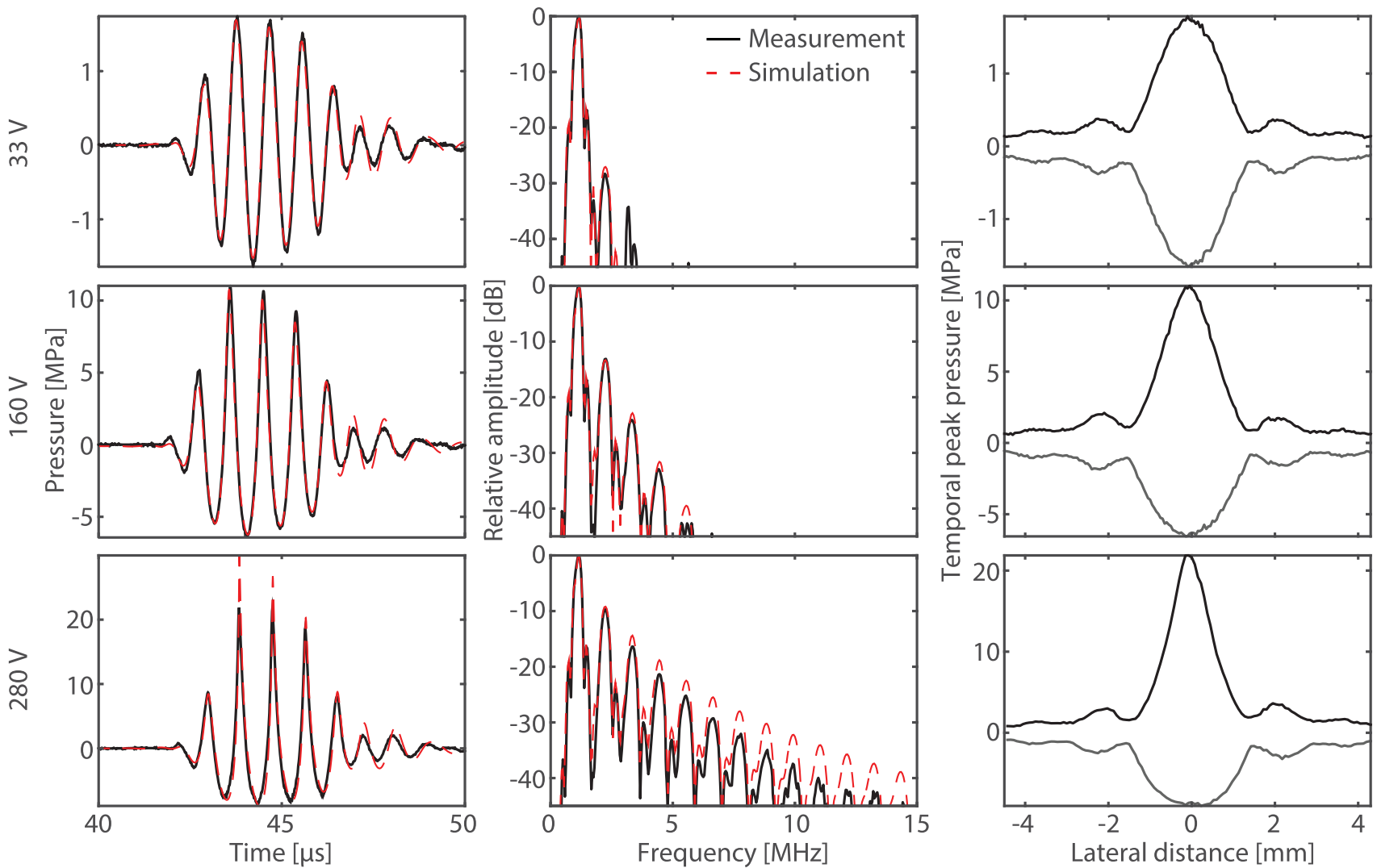

Fig. 5. Focal waveforms, spectra, and transverse peak positive and peak negative pressure profiles are shown for three different transducer drive levels. Solid lines show measured waveforms and spectra. Dashed lines show waveforms and spectra predicted from KZK simulations.

and then $\mathrm{k}$-Wave was used to back project the pressure onto the transducer surface. The waveform at a point at the center of the transducer was extracted.

The equivalent source method, used to set the boundary condition to the KZK equation on a plane to model ultrasound fields generated by strongly focused transducers [1], [6], [36], was followed. The effective source aperture, radius of curvature, and source pressure that minimized the $l_{2}$ error between the simulated axial peak pressure and that measured using a $0.2 \mathrm{~mm}$ needle hydrophone were determined. The minimization was performed using the axial peak positive pressure over a region centered on the focus of the field, including the last pre focal lobe, the focal lobe, and the beginning of the postfocal lobe. This resulted in an effective aperture diameter of $32.6 \mathrm{~mm}$ and a radius of curvature of $64.7 \mathrm{~mm}$. Differences between the effective parameters of the boundary condition to the KZK model and the actual parameters of the focused transducer arise due to the parabolic approximation of the model and setting of the boundary condition in the initial plane rather than on the spherical surface [10], [36].

It was then assumed that the source pressure linearly increased with drive voltage. This was verified by simulating the field for three different drive levels/source pressure levels for which the field had been measured with a calibrated hydrophone. The field was then simulated for each source pressure for which the field was measured.

Measurements were made with the Fabry-Pérot scanning system over a $3 \mathrm{~mm} \times 3 \mathrm{~mm}$ area covering the focal peak of the acoustic field at 19 drive levels (peak-to-peak voltage) between 16.6 and $278 \mathrm{~V}$, corresponding to source pressures of $22-360 \mathrm{kPa}$. A step size of $50 \mu \mathrm{m}$ was used and waveforms were acquired with four averages. Each scan was acquired in approximately $1.5 \mathrm{~min}$. Measurements were repeated six times at lower drive levels (up to $200 \mathrm{~V}$ ) and three times at higher drive levels.

The measured waveforms were high-pass filtered and the frequency response of the sensor was applied. The spatialpeak temporal-peak positive and negative pressures were then extracted from the data. The means of both the measured and simulated peak focal pressures are plotted in Fig. 6. Error bars show two standard deviations of the mean measured value. There is good agreement between the measured and simulated data up to drive levels of approximately $200 \mathrm{~V}$. Above this level, the simulated peak positive pressure increases more rapidly than the measured values, due to increased amplitude of harmonics in the simulation compared with the measurement, as can be observed in Fig. 5. These differences, which reach approximately $10 \%$ at the highest drive levels, could arise from several sources. Previously, good agreement was obtained between KZK simulations and measurements of a nominally identical transducer when the bandwidth of the hydrophone was applied to the simulated data [6]. Given the pressures measured here and the bandwidth of the device, the differences are not totally accounted for by the Fabry-Pérot sensor bandwidth. The effects of spatial averaging over the sensor element were also examined using the simulated data. 


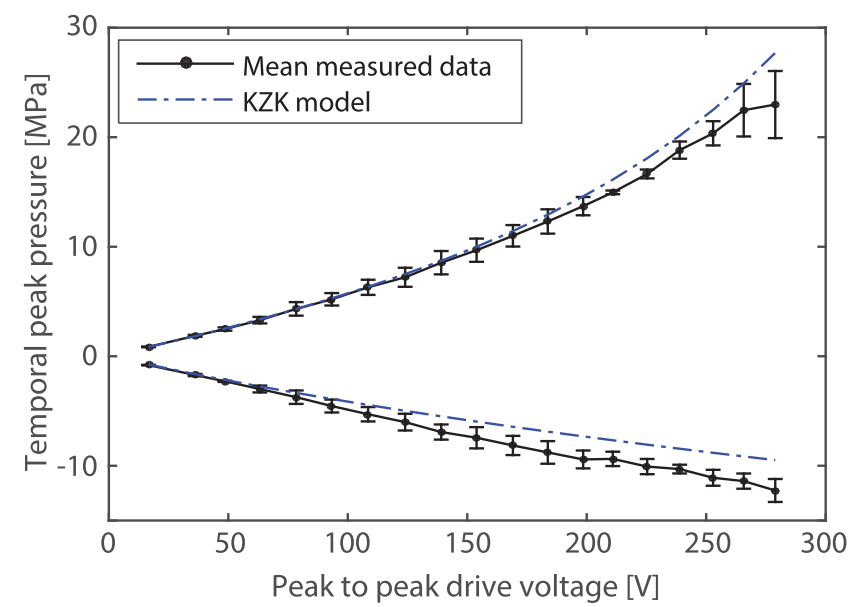

Fig. 6. Change in peak focal pressure with transducer drive level; it is assumed that the source pressure linearly changes with applied drive voltage. The solid line shows measured peak positive and negative focal pressures. Error bars show two standard deviations of the mean of three or six repeated measurements. The dotted line shows the peak positive and negative focal pressures modeled using the KZK equation.

The mean peak positive pressure was calculated over a $65 \mu \mathrm{m}^{2}$ diameter area (the effective element size) in the focal plane. At the highest drive level, there was a difference of less than $0.5 \%$ from the peak focal value, suggesting that the disagreement is not caused by spatial averaging. Another more probable reason for these differences could be the axial position of the sensor during measurements. The transducersensor distance was constant for measurements at all drive levels during a single repeat. The focus of these types of fields has been shown to move as the source pressure is increased, and due to the experimental setup, there is a possibility of small axial offsets between the sensor position and the focus. From the simulations, it was observed that for a change in axial position of $1 \mathrm{~mm}$, there was a change in peak positive pressure of approximately $5 \%$.

The smaller number of measurements at high drive levels was due to the occurrence of cavitation that disrupted beam profiles in some measurements at higher pressure. Following cavitation, there was some damage to the barrier coating on the sensor that rendered area less sensitive, but not unusable. Subsequent measurements were performed using another region of the sensor. The possibility of eliminating the barrier coating will be investigated in future work.

\section{E. Planar and Volumetric Mapping of HIFU Fields}

If a near-field planar scan is required as an input for simulation of the acoustic field, an area of at least 1.3 times the beam diameter should be scanned with spatial sampling of less than $\lambda / 2$ [23]. For the transducer used here, at a distance of $30 \mathrm{~mm}$ from the transducer origin, the beam diameter is $39 \mathrm{~mm}$. The appropriately sized $51 \mathrm{~mm} \times 51 \mathrm{~mm}$ scan plane would require $85 \times 85$ points for a step size of $0.6 \mathrm{~mm}$ $(\lambda \approx 1.4 \mathrm{~mm})$. For a hydrophone scan acquired at a rate of $1 \mathrm{~Hz}$, it would take $2 \mathrm{~h}$ to acquire, while it would take only $35 \mathrm{~s}$ to acquire with the Fabry-Pérot scanning system. While $2 \mathrm{~h}$ is not an unreasonable scan time, if the transducer was driven at

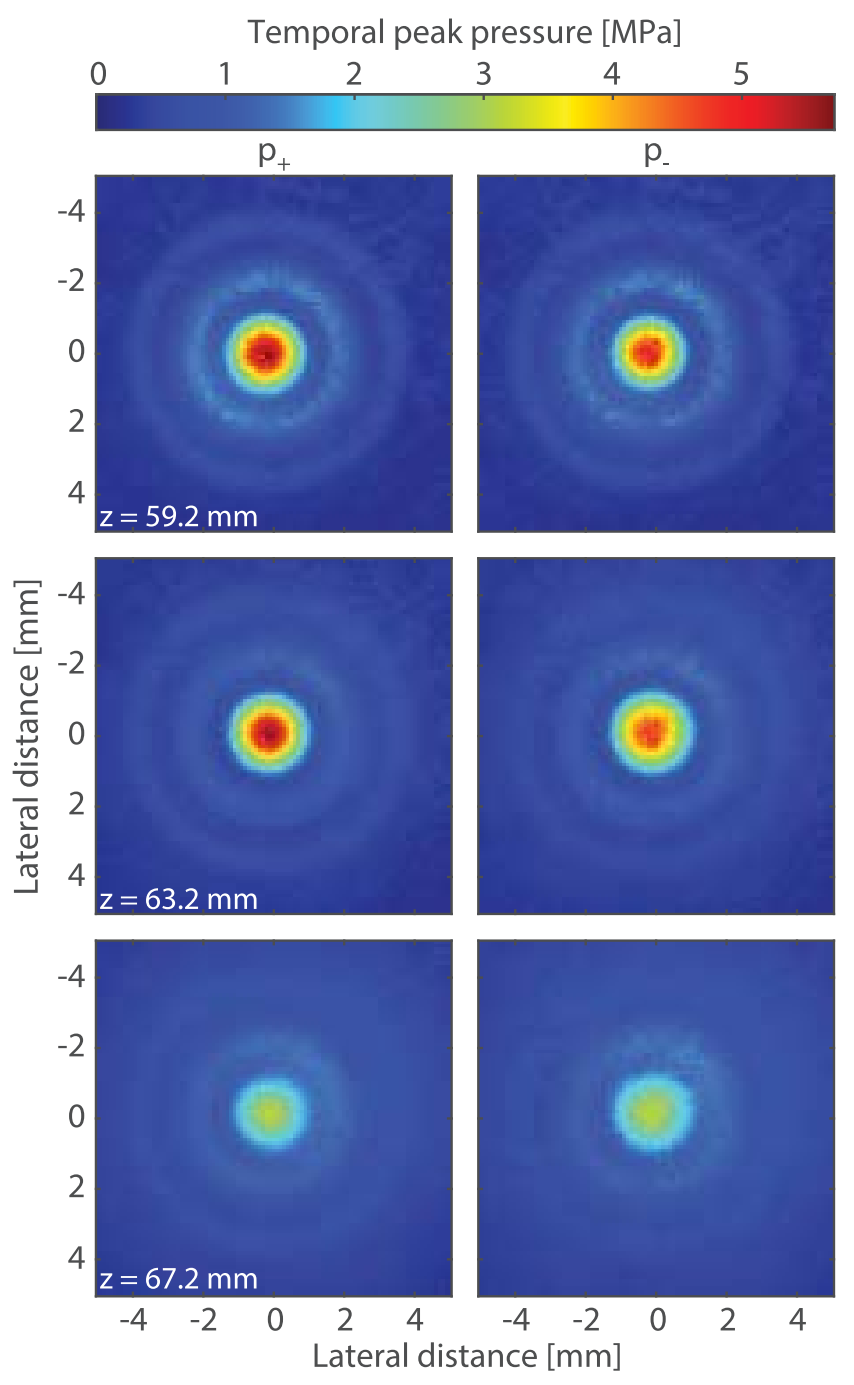

Fig. 7. Two-dimensional field maps of temporal peak positive (left) and temporal peak negative pressure (right) at $X Y$ planes positioned at distances of 59.2, 63.2 (focal plane), and $67.2 \mathrm{~mm}$ from the origin of the transducer. Scan parameters: step size $100 \mu \mathrm{m}$, scan area $10 \mathrm{~mm} \times 10 \mathrm{~mm}$, four averages, scan time $3 \mathrm{~min}$.

the third harmonic, a step size of $0.2 \mathrm{~mm}$ would be appropriate, requiring a scan plane of $255 \times 255$ points. This would take approximately $18 \mathrm{~h}$ to scan with a hydrophone, compared with approximately $5.5 \mathrm{~min}$ with the Fabry-Pérot system. With the short scan times achieved using this technique, it is also feasible to directly measure the acoustic field over a 3-D volume.

To illustrate the use of the Fabry-Pérot scanning system as a tool for rapid mapping of ultrasound fields, field scans were performed at the focal plane and on planes $4 \mathrm{~mm}$ axial distance either side of this $(59.2,63.2$, and $67.2 \mathrm{~mm}$ from the source). The transducer to sensor distance was adjusted using a manual translation stage. The transducer was driven with a peak-to-peak voltage of $94 \mathrm{~V}$ and an area of $10 \mathrm{~mm} \times 10 \mathrm{~mm}$ was scanned with a step size of $100 \mu \mathrm{m}(100 \times 100$ points $)$ with four averages. The scan time was approximately $3 \mathrm{~min}$ per plane.

Plots of the peak positive and negative pressure on each plane are shown in Fig. 7. The spatial-peak temporal-peak 
TABLE I

Characteristics of Hydrophones ApPlied to MeAsurement of Focused Ultrasound Fields

\begin{tabular}{|c|c|c|c|c|c|c|}
\hline \multirow[b]{2}{*}{ Device type } & \multirow{2}{*}{$\begin{array}{l}\text { Element diameter } \\
\qquad[\mu m]\end{array}$} & \multirow{2}{*}{$\begin{array}{c}-6 \mathrm{~dB} \text { bandwidth } \\
{[\mathrm{MHz}]}\end{array}$} & \multicolumn{2}{|c|}{ Highest reported pressure } & \multirow{2}{*}{$\begin{array}{c}\text { NEP (type, bandwidth) } \\
{[\mathrm{kPa}]}\end{array}$} & \multirow{2}{*}{ Reference } \\
\hline & & & $\begin{array}{c}p_{+} \\
{[\mathrm{MPa}]}\end{array}$ & $\begin{array}{c}p_{-} \\
{[\mathrm{MPa}]}\end{array}$ & & \\
\hline Planar hard dielectric Fabry-Pérot sensor & 65 & $80+$ & 25 & 12 & $49-152(3 \sigma, 125 \mathrm{MHz})$ & \\
\hline Protected needle hydrophone & 400 & 10 & 25 & 9 & $130(\sigma, 20 \mathrm{MHz})$ & [7], [8] \\
\hline Conventional membrane hydrophone & 200 & 100 & 27 & 10 & $13\left(\sigma^{2}, 500 \mathrm{MHz}\right)$ & [3] \\
\hline Protected membrane hydrophone & 200 & $40+$ & 75 & 15 & $65\left(\sigma^{2}, 500 \mathrm{MHz}\right)$ & [3] \\
\hline Eisenmenger type fibre-optic hydrophone & 100 & 100 & $100+$ & 18 & $2000-3000(100 \mathrm{MHz})$ & {$[2]$} \\
\hline
\end{tabular}

positive pressure at the focal plane was $5.6 \mathrm{MPa}$, while the spatial-peak temporal-peak negative pressure was $4.7 \mathrm{MPa}$. Side lobes are visible on each of the planes, and differences in their amplitude can be seen as the axial distance is varied. The smallest clearly visible side lobes have an amplitude of approximately $600 \mathrm{kPa}$. For speed, all three scans were performed with the same interrogation laser power. The NEP for this pressure range was $47 \mathrm{kPa}(20 \mathrm{MHz}$ bandwidth) or $80 \mathrm{kPa}$ (125 MHz bandwidth). To maximize the SNR, the laser power could also be adjusted from plane to plane or from point to point.

\section{CONCLUSION}

A Fabry-Pérot sensor of all hard dielectric construction was developed and characterized in terms of its frequency response, directivity, and NEP and used to make measurements of a nonlinear focused ultrasound field. The main characteristics of the system are reported in Table I, along with the characteristics of other devices applied to measurement of high-amplitude focused ultrasound fields. Its suitability and limitations for application to measurement of high acoustic pressure nonlinear focused ultrasound fields are discussed here.

The main advantage of the system is the acquisition speed. Waveforms were acquired from 200 scan points each second; this is very rapid compared with the usual rate of around 1 scan point per second for conventional hydrophone scans. The acquisition time for each of the $10 \mathrm{~mm} \times 10 \mathrm{~mm}$ scan planes shown in Fig. 7 was approximately 3 min, whereas the equivalent hydrophone scans would take almost $3 \mathrm{~h}$ each.

Another advantage of the sensor is the small element size of $65 \mu \mathrm{m}$, the smallest of the sensors listed in Table I. While this is an advantage in reducing spatial averaging in the increasingly narrow focal regions of high amplitude fields, it has been shown that greater error in measured focal pressure can arise if the sensor bandwidth is limited [6]. For this sensor, the on-axis amplitude frequency response was constant to within $3 \mathrm{~dB}$ up to $62 \mathrm{MHz}$ and to within less than $6 \mathrm{~dB}$ up to $80 \mathrm{MHz}$, which was the limit of the frequency content of the acoustic field used to measure the frequency response. The bandwidth is greater than the protected needle and membrane hydrophones (although their effective bandwidth was increased by deconvolution of the complex frequency response) and theoretically, the frequency response is expected to be constant to frequencies of over $100 \mathrm{MHz}$. This would make the bandwidth comparable with a conventional membrane hydrophone or FOPH and suitable for measurement nonlinear fields containing many harmonics.

The NEP of the system scaled with the pressure measurement range, by adjustment of the interrogating laser power. At the low end of the measurement range (up to $8 \mathrm{MPa}$ ), the NEP was $26 \mathrm{kPa}$ over a $20 \mathrm{MHz}$ bandwidth and $49 \mathrm{kPa}$ over the full $125 \mathrm{MHz}$ bandwidth. This compares well with a PVDF needle hydrophone of a similar element size $(75 \mu \mathrm{m})$ for which the NEP has been quoted as $50 \mathrm{kPa}$ (20 MHz bandwidth) [14]. The NEP for the highest pressures measured in this paper was $152 \mathrm{kPa}$, which is higher than both the conventional and the protected membrane hydrophone but considerably lower than the NEP on the Eisenmenger-type FOPH.

In this paper, spatial mapping of a focused ultrasound field was achieved with maximum peak positive and negative focal pressures of 25 and $-12 \mathrm{MPa}$, respectively. While lower than the $75 \mathrm{MPa}$ peak positive pressure and $15 \mathrm{MPa}$ peak negative pressure measured using the protected membrane hydrophones [3] and the 100+ MPa peak positive and $18 \mathrm{MPa}$ peak negative pressures measure using the Eisenmenger-type FOPH [2], these pressure levels are comparable with the highest reported pressures measured with a conventional membrane hydrophone and protected needle hydrophone. For these three devices, the pressure levels were limited by the onset of cavitation that disrupted the measurements. The PVDF hydrophones suffered damaged to their electrodes, which prevented further use. The Fabry- Pérot sensor suffered some damage to the barrier coating rather than the interferometer itself. This damage then made cavitation more likely, probably due to increased surface roughness. The possibility of manufacturing sensors without the barrier coating will be investigated in future work. In most cases, exposure of expensive PVDF hydrophones to high pressures would be avoided because of the risk of damage. However, replacement of the Fabry-Pérot sensor head is simple and much less costly so could provide an alternative method of measuring pressure at these levels without risk of damage to expensive equipment.

There are several features of the sensor and the experimental set up for this system that increase the likelihood of cavitation. Reflection of the acoustic field at the large water-sensor interface causes an approximate doubling of the 
acoustic pressure at the sensor. Because of this, the maximum pressure that can be measured before cavitation occurs will be approximately half of the intrinsic cavitation threshold of deionized water, which is on the order of 24-28 $\mathrm{MPa}$ at room temperature [37]. During measurements, cavitation always occurred when the peak negative pressure exceeded a level of approximately $13 \mathrm{MPa}$. In practice, cavitation was also likely below this threshold because of reoxygenation and contamination of the relatively small volume of water in the tank. The sensor also has a large surface area, creating more locations for cavitation nuclei, and it was mounted in the base of the tank so that impurities could settle onto the surface and act as cavitation nuclei. These factors are not an issue when, for example, a small FOPH is used in a large water tank, or with a hydrophone specifically designed to suppress cavitation at the sensitive element.

Comparison with hydrophone measurements at low drive levels showed some discrepancies due to the directional response of the Fabry-Pérot sensor. There was good agreement in the focal lobe of the field, but a higher pressure amplitude was measured in the side lobes. The differences were significantly reduced by deconvolution of the directional response from the measured data. However, application of the deconvolution to the measured data is complicated in practice, due to the low sensitivity at some angles of incidence, and will be the subject of further work. Uncertainties in the measurement of the directional response, which could arise from misalignments during its measurement, will propagate through the deconvolution. This is an extra source of uncertainty that is minimal for conventional hydrophones with small element sizes. Another source of uncertainty was the low amplitude of the laser generated ultrasound field used for measurement of on axis frequency response. This could be improved by optimization of the laser generated ultrasound source to increase its amplitude, or by adapting the mounting of the sensor so a more traditional hydrophone calibration method could be used. At higher drive levels, measurements of focal pressure were compared with KZK simulations. There was good agreement for drive levels of up to about $200 \mathrm{~V}$ $\left(p_{0}=260 \mathrm{kPa}\right)$, but above this level, the measured peak positive pressure was lower than the simulated value. It seems likely that this was caused at least partly by misalignment of the sensor on the beam axis.

In this paper, the transducer was driven with a short pulse, rather than a longer tone burst reaching steady-state conditions that would be more representative of a clinical HIFU driving regime. The reason for this was the limited measurement time before interference from reflections from the back of the sensor substrate. In future iterations of the sensor design, the interferometer will be deposited onto a thicker substrate to allow time for measurement of longer toneburst signals.

The measured pressures did not exceed the linear measurement range of the sensor, which was $35 \mathrm{MPa}$ to within 5\% and $56 \mathrm{MPa}$ to within $10 \%$. To enable measurement of higher pressures, provided that cavitation could be avoided, this could be increased by reducing the finesse of the interferometer by decreasing the reflectivity of the mirrors or reducing the spacer thickness.
In conclusion, the rapid scanning time, small element size, reasonably low NEP, and flat and broad frequency response of the sensor are advantages in the measurement of nonlinear focused ultrasound fields. However, there are currently some limitations in terms of the maximum pressure amplitude and onset of cavitation, and the linear range and free measurement time of the sensor. Overcoming these limitations to extend the measurements to clinical HIFU fields will be investigated as part of future work.

\section{REFERENCES}

[1] M. S. Canney, M. R. Bailey, L. A. Crum, V. A. Khokhlova, and O. A. Sapozhnikov, "Acoustic characterization of high intensity focused ultrasound fields: A combined measurement and modeling approach," J. Acoust. Soc. Amer, vol. 124, no. 4, pp. 2406-2420, 2008.

[2] W. Kreider et al., "Characterization of a multi-element clinical HIFU system using acoustic holography and nonlinear modeling," IEEE Trans. Ultrason., Ferroelect., Freq. Control, vol. 60, no. 8, pp. 1683-1698, Aug. 2013.

[3] V. Wilkens, S. Sontag, and O. Georg, "Robust spot-poled membrane hydrophones for measurement of large amplitude pressure waveforms generated by high intensity therapeutic ultrasonic transducers," J. Acoust. Soc. Amer., vol. 139, no. 3, pp. 1319-1332, 2016.

[4] A. Hurrell and P. Beard, "Piezoelectric and fibre-optic hydrophones," in Ultrasonic Transducers, K. Nakamura, Ed. Oxford, U.K.: Woodhead Publishing, 2012, pp. 621-622.

[5] S. P. Robinson, "Hydrophones," in Output Measurements for Medical Ultrasound, R. C. Preston, Ed. London, U.K.: Springer-Verlag, 1991, ch. 4 , p. 62 .

[6] O. V. Bessonova and V. Wilkens, "Membrane hydrophone measurement and numerical simulation of HIFU fields up to developed shock regimes," IEEE Trans. Ultrason., Ferroelect., Freq. Control, vol. 60, no. 2, pp. 290-300, Feb. 2013.

[7] S. M. Howard and C. I. Zanelli, "Characterization of a HIFU field at high intensity," in Proc. IEEE Ultrason. Symp., Oct. 2007, pp. 1302-1304.

[8] J. Haller et al., "A comparative evaluation of three hydrophones and a numerical model in high intensity focused ultrasound fields," J. Acoust. Soc. Amer., vol. 131, no. 2, pp. 1121-1130, 2012.

[9] J. Staudenraus and W. Eisenmenger, "Fibre-optic probe hydrophone for ultrasonic and shock-wave measurements in water," Ultrasonics, vol. 31, no. 4, pp. 267-273, 1993.

[10] P. B. Rosnitskiy et al., "Design of HIFU transducers for generating specified nonlinear ultrasound fields," IEEE Trans. Ultrason., Ferroelect., Freq. Control, vol. 64, no. 2, pp. 374-390, Feb. 2017.

[11] J. Haller et al., "Characterization of a fiber-optic displacement sensor for measurements in high-intensity focused ultrasound fields," J. Acoust. Soc. Amer, vol. 129, no. 6, pp. 3676-3681, 2011.

[12] P. Morris et al., "A Fabry-Pérot fiber-optic ultrasonic hydrophone for the simultaneous measurement of temperature and acoustic pressure," J. Acoust. Soc. Amer, vol. 125, no. 6, pp. 3611-3622, 2009.

[13] E. Zhang, J. Laufer, and P. Beard, "Backward-mode multiwavelength photoacoustic scanner using a planar Fabry-Pérot polymer film ultrasound sensor for high-resolution three-dimensional imaging of biological tissues," Appl. Opt., vol. 47, no. 4, pp. 561-577, 2008.

[14] P. C. Beard, A. M. Hurrell, and T. N. Mills, "Characterization of a polymer film optical fiber hydrophone for use in the range 1 to $20 \mathrm{MHz}$ : A comparison with PVDF needle and membrane hydrophones," IEEE Trans. Ultrason., Ferroelect., Freq. Control, vol. 47, no. 1, pp. 256-264, Jan. 2000.

[15] V. Wilkens, "Characterization of an optical multilayer hydrophone with constant frequency response in the range from 1 to $75 \mathrm{MHz}$," J. Acoust. Soc. Amer., vol. 113, no. 3, pp. 1431-1438, Mar. 2003.

[16] V. Wilkens and C. Koch, "Fiber-optic multilayer hydrophone for ultrasonic measurement," Ultrasonics, 37, no. 1, pp. 45-49, 1999.

[17] V. Wilkens and C. Koch, "Optical multilayer detection array for fast ultrasonic field mapping," Opt. Lett., vol. 24, no. 15, pp. 1026-1028, 1999.

[18] V. Wilkens and C. Koch, "Amplitude and phase calibration of hydrophones up to $70 \mathrm{MHz}$ using broadband pulse excitation and an optical reference hydrophone," J. Acoust. Soc. Amer., vol. 115, no. 6, pp. 2892-2903, Jun. 2004.

[19] Y. Jing et al., "Experimental verification of transient nonlinear acoustical holography," J. Acoust. Soc. Amer., vol. 133, no. 5, pp. 2533-2540, 2013. 
[20] Ultrasonics-Hydrophones: Part 1: Measurement and Characterization of Medical Ultrasonic Fields up to $40 \mathrm{MHz}$, document IEC 621271:2007+A1:2013, Int. Electrotech. Commission, Geneva, Switzerland, 2013.

[21] Ultrasonics - Field characterization-Specification and Measurement of Field Parameters for High Intensity Therapeutic Ultrasound (HITU) Transducers and Systems, document IEC TS 62556:2104, Int. Electrotech. Commission, Geneva, Switzerland 2014.

[22] E. Martin, Y. T. Ling, and B. E. Treeby, "Simulating focused ultrasound transducers using discrete sources on regular Cartesian grids," IEEE Trans. Ultrason., Ferroelect., Freq. Control, vol. 63, no. 10, pp. 1535-1542, Oct. 2016.

[23] O. A. Sapozhnikov, S. A. Tsysar, V. A. Khokhlova, and W. Kreider, "Acoustic holography as a metrological tool for characterizing medical ultrasound sources and fields," J. Acoust. Soc. Amer., vol. 138, no. 3, pp. $1515-1532,2015$

[24] S. Oyama, J. Yasuda, H. Hanayama, S. Yoshizawa, and S.-I. Umemura, "Quantitative measurement of ultrasound pressure field by optical phase contrast method and acoustic holography," Jpn. J. Appl. Phys., vol. 55, no. 7S1, p. 07KB09, 2016.

[25] E. Zhang, P. A. Beard, "Broadband ultrasound field mapping system using a wavelength tuned, optically scanned focused laser beam to address a Fabry Perot polymer film sensor," IEEE Trans. Ultrason. Ferroelect., Freq. Control, vol. 53, no. 7, pp. 1330-1338, Jul. 2006.

[26] M. Klann and C. Koch, "Measurement of spatial cross sections of ultrasound pressure fields by optical scanning means," IEEE Trans. Ultrason., Ferroelect., Freq. Control, vol. 52, no. 9, pp. 1546-1554, Sep. 2005

[27] B. E. Treeby, B. T. Cox, E. Z. Zhang, S. K. Patch, and P. C. Beard, "Measurement of broadband temperature-dependent ultrasonic attenuation and dispersion using photoacoustics," IEEE Trans. Ultrason., Ferroelect., Freq. Control, vol. 56, no. 8, pp. 1666-1676, Aug. 2009.

[28] P. C. Beard, F. Pérénnes, and T. N. Mills, "Transduction mechanisms of the Fabry-Perot polymer film sensing concept for wideband ultrasound detection," IEEE Trans. Ultrason., Ferroelect., Freq. Control, vol. 46, no. 6, pp. 1575-1582, Nov. 1999.

[29] J. A. Guggenheim, E. Z. Zhang, and P. C. Beard, "A method for measuring the directional response of ultrasound receivers in the range $0.3 \mathrm{MHz}$ to $80 \mathrm{MHz}$ using a laser generated ultrasound source," IEEE Trans. Ultrason., Ferroelect., Freq. Control, to be published.

[30] T. H. Laby and G. W. C. Kaye, "The speed and attenuation of sound," in Tables of Physical \& Chemical Constants, 16th ed. Teddington, U.K.: Kaye Laby Online, 2005, sec. 2.4.1. [Online]. Available: http://www.kayelaby.npl.co.uk/

[31] H.-S. Noh, K.-S. Moon, A. Cannon, P. J. Hesketh, and C. P. Wong, "Wafer bonding using microwave heating of parylene intermediate layers," J. Micromech. Microeng., vol. 14, no. 4, pp. 625-631, 2004.

[32] P. C. Beard and T. N. Mills, "Extrinsic optical-fiber ultrasound sensor using a thin polymer film as a low-finesse Fabry-Perot interferometer," Appl. Opt., vol. 35, no. 4, pp. 663-675, 1996.

[33] V. Wilkens, C. Wiemann, C. Koch, and H.-J. Foth, "Fiber-optic dielectric multilayer temperature sensor: In situ measurement in vitreous during Er:YAG laser irradiation," Opt. Laser Technol., vol. 31, no. 8, pp. 593-599, 1999.

[34] (Mar. 2001). Focused Beams: KZK Texas Time Domain Code. Accessed: Sep. 13, 2016. [Online]. Available: http://people.bu.edu/robinc/kzk/ focus.html

[35] M. A. Averkiou and R. O. Cleveland, "Modeling of an electrohydraulic lithotripter with the KZK equation," J. Acoust. Soc. Amer., vol. 106 , no. 1, pp. 102-112, 1999.

[36] P. B. Rosnitskiy, P. V. Yuldashev, B. A. Vysokanov, and V. A. Khokhlova, "Setting boundary conditions on the Khokhlov-Zabolotskaya equation for modeling ultrasound fields generated by stongly focused transducers," Acoust. Phys, vol. 62, no. 2, pp. 151-159, 2016.

[37] E. Herbert, S. Balibar, and F. Caupin, "Cavitation pressure in water," Phys. Rev. E, Stat. Phys. Plasmas Fluids Relat. Interdiscip. Top., vol. 74, no. 4, p. 041603, 2006.

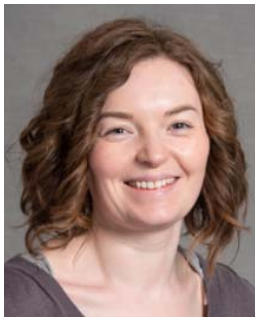

Eleanor Martin was born in Newcastle upon Tyne, U.K. She received the M.Sc. degree (Hons.) in physics with astrophysics from the University of Bristol, Bristol, U.K., in 2004, and the M.Sc. degree in medical physics and the Ph.D. degree in physics from the University of Exeter, Exeter, U.K., in 2006 and 2010, respectively.

She was a Higher Research Scientist with the Acoustics Group, Medical Ultrasonics Section, National Physical Laboratory, Teddington, U.K., from 2011 to 2014, where she was involved in ultrasound metrology. She moved to the Biomedical Ultrasound Group, Department of Medical Physics and Biomedical Engineering, University College London, London, U.K., in 2014, where she is currently a Post-Doctoral Research Associate. Her current research interests include the experimental validation of models of nonlinear ultrasound propagation and the measurement of acoustic fields at high pressure levels.

Edward Z. Zhang, photograph and biography not available at the time of publication.

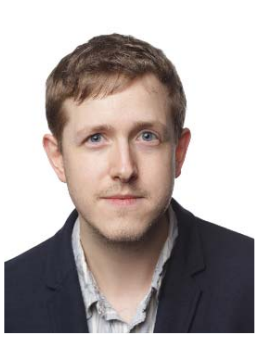

James A. Guggenheim was born in Birmingham, U.K. He received the B.Sc. and M.Sc. degrees in artificial intelligence and computer science and Intelligent Systems Engineering from the University of Birmingham, Birmingham, in 2008 and 2009, respectively, and the M.Sc. degree and the Ph.D. degree, in topics related to biomedical imaging, from the Physical Science of Imaging in Biomedical Sciences Doctoral Training Centre, University of Birmingham, in 2010 and 2014, respectively.

Since 2013, he has been a Research Associate in biomedical photoacoustic imaging instrumentation with the Department of Medical Physics and Biomedical Engineering, University College London, London, U.K. His current research interests include photoacoustic imaging and spectroscopy, ultrasensitive optical ultrasound sensors, and ultrasonic and photoacoustic system characterization.

Paul C. Beard, photograph and biography not available at the time of publication.

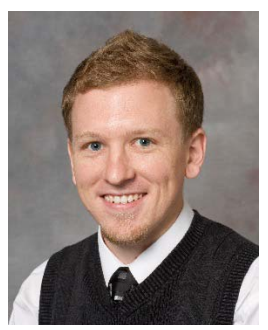

Bradley E. Treeby (M'13) was born in Albany, Australia. He received the B.E. degree (Hons.) in mechatronics engineering and the Ph.D. degree in acoustics and mechanical engineering from The University of Western Australia, Perth, WA, Australia, in 2003 and 2007, respectively.

$\mathrm{He}$ is currently an EPSRC Early Career Fellow with the Department of Medical Physics and Biomedical Engineering, University College London, London, U.K. He has authored over 60 scientific papers. He has co-authored an open-source acoustics toolbox for MATLAB called k-Wave. His current research interests include biomedical ultrasound, numerical methods, and high performance computing. Dr. Treeby is a member of the Acoustical Society of America. 